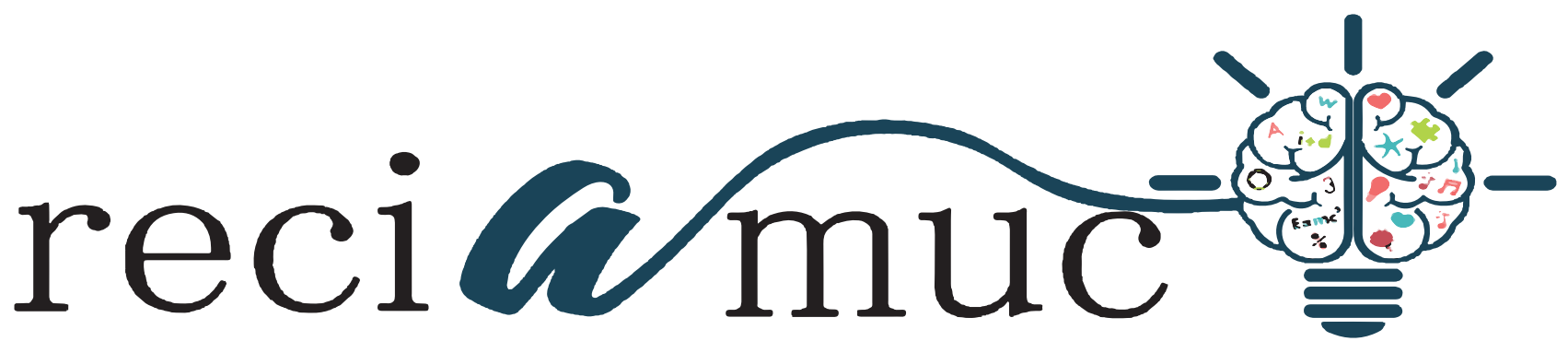

DOI: 10.26820/reciamuc/6.(1).enero.2022.64-71

URL: https://reciamuc.com/index.php/RECIAMUC/article/view/783

EDITORIAL: Saberes del Conocimiento

REVISTA: RECIAMUC

ISSN: 2588-0748

TIPO DE INVESTIGACIÓN: Artículo de Investigación

Código UNESCO: 32 Ciencias Médicas

PAGINAS: 64-71

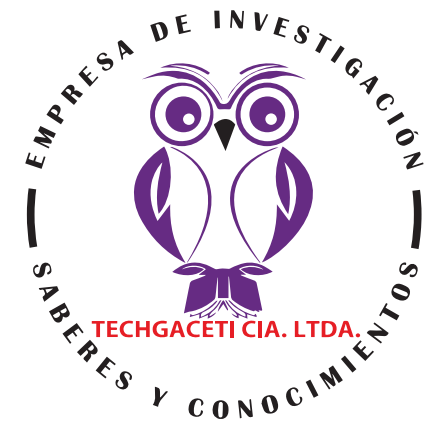

\title{
Pancreatitis aguda en pacientes con litiasis biliar ingresados en el hospital Martín Icaza
}

Acute pancreatitis in patients with gallstones admitted to the Martín Icaza hospital

Pancreatite aguda em pacientes com cálculos biliares internados no hospital Martín Icaza

Madelyne Valeria López Díaz'; Hugo Fernando Cabrera Bernal2;
Ana María Viteri Rojas ${ }^{3}$; María Paulina Reyes Prieto Aguayo ${ }^{4}$

RECIBIDO: 15/11/2021 ACEPTADO: 05/12/2021 PUBLICADO: 30/01/2022

1. Médico de la Universidad de Guayaquil; Investigador Independiente; Guayaquil, Ecuador; madelyne96@hotmail.com; (ID https://orcid.org/0000-0003-4500-9634

2. Médico de la Universidad de Guayaquil; Investigador Independiente; Guayaquil, Ecuador; hugo-1696@hotmail.com; (D) https://orcid.org/0000-0003-4758-697X

3. Especialista en Cirugía General; Doctora en Medicina y Cirugía; Docente de la Facultad de Ciencias Médicas, Carrera de Medicina; Universidad de Guayaquil, Ecuador; anyviteri031@hotmail.com; (D) https://orcid. org/0000-0002-5279-0089

4. Médico de la Universidad de Guayaquil; Investigador Independiente; Guayaquil, Ecuador; maripare_12@hotmail.com; (D) https://orcid.org/0000-0002-5315-4869

CORRESPONDENCIA

Madelyne Valeria López Díaz

madelyne96@hotmail.com

Guayaquil, Ecuador

๑) RECIAMUC; Editorial Saberes del Conocimiento, 2022 


\section{RESUMEN}

La pancreatitis aguda se produce comúnmente por la obstrucción que genera los cálculos biliares lo cual da origen a la autodigestión de la glándula por sus propias enzimas. Se caracteriza por dolor abdominal, náuseas, vómitos, y predomina en el sexo femenino. El objetivo del presente estudio es analizar la pancreatitis aguda en pacientes con litiasis biliar ingresados en el Hospital General Martin Icaza durante el año 2020 - 2021. Es un estudio retrospectivo, no experimental de corte transversal, descriptivo y analítico debido al análisis de las historias clínicas de los pacientes ingresados en el Hospital General Martin Icaza 2020 - 2021. Durante el periodo comprendido entre el 1 enero del 2020, hasta el 1 de mayo del 2021 se detectaron 31 pacientes con pancreatitis aguda de origen biliar, donde se encontró que el $36 \%$ de casos se presentó a los 20-25 años de edad; siendo más común la población femenina con un $65 \%$; también se detectó que los pacientes acuden a la unidad hospitalaria por presentar dolor abdominal 30\% y vomito $25 \%$, y que el $16 \%$ presentaba antecedentes patológicos personales de litiasis biliar. En base a este estudio podemos concluir que el síntoma más característico es el dolor abdominal, las edades más frecuentes en desarrollar una pancreatitis aguda oscila entre los 20 a 25 años, el sexo más predominante en desarrollar pancreatitis aguda es el femenino, el factor de riesgo más frecuente es la de origen biliar.

Palabras clave: Pancreatitis aguda, litiasis biliar, salud, factores de riesgo.

\section{ABSTRACT}

Acute pancreatitis is commonly caused by obstruction caused by gallstones, which gives rise to self digestion of the gland by its own enzymes. It is characterized by abdominal pain, nausea, vomiting, and predominates in women. The main objective of this research is to analyze acute pancreatitis in patients with gallstones admitted to the General Hospital Martin Icaza during the year 2020 - 2021 . It is a retrospective, non-experimental, cross-sectional, descriptive and analytical study due to the analysis of medical records of patients admitted to the Martin Icaza General Hospital between January 1, 2020, and May 1, 2021. 31 patients with acute pancreatitis of biliary origin were detected, where it was found that $36 \%$ of cases presented at $20-25$ years of age; being more common the female population with $65 \%$; It was also detected that the patients came to the hospital with $30 \%$ abdominal pain and $25 \%$ vomiting, and that $16 \%$ had a personal medical history of gallstones. Based on this research we can conclude that the most characteristic symptom is abdominal pain, the most frequent ages to develop acute pancreatitis ranges from 20 to 25 years, the most predominant gender in developing acute pancreatitis is female, the more frequent risk factor is the biliary origin.

Keywords: Acute pancreatitis, gallstones, health, prevention, wellness.

\section{RESUMO}

A pancreatite aguda é comumente causada por obstrução causada por cálculos biliares, o que dá origem à autodigestão da glândula por suas próprias enzimas. Caracteriza-se por dor abdominal, náuseas, vômitos e predomina em mulheres. O objetivo principal desta pesquisa é analisar a pancreatite aguda em pacientes com cálculos biliares internados no Hospital Geral Martin Icaza durante o ano de 2020 - 2021. É um estudo retrospectivo, não experimental, transversal, descritivo e analítico devido à análise de prontuários de pacientes internados no Hospital Geral Martin Icaza entre $1^{\circ}$ de janeiro de 2020 e $1^{\circ}$ de maio de 2021 . Foram detectados 31 pacientes com pancreatite aguda de origem biliar, onde se verificou que 36\% dos casos apresentavam-se entre $20-25$ anos de idade; sendo mais comum a população feminina com $65 \%$; Também foi detectado que os pacientes chegaram ao hospital com $30 \%$ de dor abdominal e $25 \%$ de vômitos, e que 16\% tinham histórico médico pessoal de cálculos biliares. Com base nesta pesquisa podemos concluir que o sintoma mais característico é a dor abdominal, a idade mais frequente para desenvolver pancreatite aguda varia de 20 a 25 anos, o gênero mais predominante no desenvolvimento de pancreatite aguda é o feminino, o fator de risco mais frequente é o biliar origem.

Palavras-chave: Pancreatite aguda, cálculos biliares, saúde, prevenção, bem-estar. 


\section{Introducción}

La pancreatitis aguda es una de las patologías más frecuentes que requieren ingreso hospitalario en todo el mundo con una incidencia anual es de 13- 45 casos por cada 100.000 personas. De acuerdo al país, el sexo y factor desencadenante varia su etiología. En Europa y Latinoamérica se demostró que se encuentra asociada, en la mayoría de los casos a litiasis biliar mientras que en Estados Unidos, países escandinavos y Brasil la etiología más frecuente es la alcohólica. (Brito, 2002)

Según el INEC, en Ecuador en el año 2019 se detectaron 5.478 casos de pancreatitis aguda a nivel nacional de los cuales, 1.247 se encuentran en la provincia del Pichincha, 1.092 en la provincia del Guayas a diferencia de la provincia de Los Ríos donde solo se detectaron 170 casos; también en base a sus estadísticas pudimos analizar que esta patología es más frecuente en mujeres mayores de 65 años y que la tasa de letalidad se redujo a de $2.12 \%$ en relación al año 2016 donde hubo un total de 141 fallecidos con una tasa de letalidad de $2.99 \%$. (Instituto Nacional de Estadísticas, 2019)

La falta de conocimiento de los pacientes entre 20 a 50 años de edad sobre el desarrollo de esta enfermedad, como de los factores de riesgo ha generado gran interés por parte del personal del salud; debido a la reacción inflamatoria brusca del páncreas producidas principalmente por la obstrucción de la ampolla de Vater debido a la presencia de litiasis biliar, seguido del consumo excesivo de alcohol y entre otras causas aunque poco comunes como la intoxicación medicamentosa que se presenta en el 1,4 a $2 \%$ de los casos (Ledesma-Heyer \& Arias Amaral, 2009) (Jean-Louis Frossard 1, 2008), la hipertrigliceridemia, la hipercalcemia, obstrucciones de la salida del conducto del páncreas, golpes en el abdomen, algunas intervenciones quirúrgicas como de la colangiopancreatografía retrógrada endoscópica (CPRE) que también pueden producir pancreatitis aguda; se han dado casos que en el $20 \%$ de los pacientes con pancreatitis aguda suele ser de origen idiopático, de los cuales el 5\% sólo sufren nuevos episodios de pancreatitis. (Junquera \& Pereyra, 2010)

El cuadro clínico de esta patología se caracteriza por la presencia de dolor agudo e intenso en el epigastrio que se puede irradiar en banda hacia los flancos (50\% de los casos), o acompañarse de náuseas y vómitos en $90 \%$ de los casos. (Frossard, Steer, \& Pastor, 2008) (Swaroop, Chari, \& Clain, 2004) se han detectado que en el 5 a 10\% de los pacientes presentan una pancreatitis indolora siendo más común en pacientes bajo diálisis peritoneal o en postrasplantados de riñón. (Toouli, Brooke-Smith, \& Bassi, 2002) son de peor pronostico los casos de los pacientes que presentan signos clínicos como grey-turner y de cullen aunque esto no es patonogmotico del desarrollo de una pancreatitis hemorrágica. (Frossard, Steer, \& Pastor, 2008)

Generalmente los casos de pancreatitis leve responden a un manejo conservador, mientras q las formas mas graves tienen una morbilidad y mortalidad significativa.

La pancreatitis aguda es una enfermedad con más ingresos hospitalarios en nuestro medio; es por esta razón que esta investigación tiene como propósito principal, analizar el desarrollo de pancreatitis aguda en pacientes con litiasis biliar; determinando factores de riesgo, sexo, así como la edad más frecuente en la que esta se manifestó en pacientes que han sido ingresados en el Hospital General Martín Icaza del cantón Babahoyo provincia de Los Ríos, durante el año 2020-2021; de esta manera, contribuimos al diagnóstico precoz y elección de tratamiento adecuado para mejorar el estilo de vida de los pacientes que desarrollaron pancreatitis aguda y quienes aún no la padecen. 


\section{Metodología}

El presente estudio es realizado con un enfoque cuantitativo debido a la implementación de una base datos de los pacientes con diagnóstico de pancreatitis aguda por litiasis biliar (CIE 10: K85 PANCREATITIS AGUDA). Es un estudio retrospectivo, no experimental de corte transversal, descriptivo y analítico debido al análisis de las historias clínicas de los 46 pacientes ingresados en el Hospital General Martin Icaza durante el periodo comprendido entre el 1 enero del 2020, hasta el 1 de mayo del 2021.

Se tomó una muestra de 31 pacientes que cumplían con los criterios de inclusión y exclusión; en esta investigación no se aplicó formula estadística para determinar la muestra de estudio debido a que se trabajó la totalidad de las historias disponibles. La información se recolecta mediante la revisión de las historias clínicas de las carpetas del departamento de estadística del hospital, en los cuales se detallan antecedentes personales y familiares, evolución, diagnóstico y tratamiento realizado. Se elaboró un documento en Excel donde se organizó la información mediante la observación directa y utilizando un método cuantitativo de todos los pacientes.

\section{Resultados}

Luego de recabar la información obtenida de la base de datos otorgada por el departamento estadístico del Hospital General Martin Icaza se logró determinar mediante nuestra hoja de recopilación de datos que:

Tabla 1. Casos de Pancreatitis aguda según el grupo etario.

\begin{tabular}{|c|c|c|}
\hline \multicolumn{3}{|c|}{ Pancreatitis aguda según el grupo etario } \\
\hline Edad en años & $\mathbf{N}^{\circ}$ & $\%$ \\
\hline $20-25$ años & 11 & $36 \%$ \\
\hline 26 a 30 años & 6 & $19 \%$ \\
\hline 31 a 35 años & 4 & $13 \%$ \\
\hline 36 a 40 años & 1 & $3 \%$ \\
\hline 41 a 45 años & 2 & $6 \%$ \\
\hline 46 a 50 años & 7 & $23 \%$ \\
\hline total & 31 & $100 \%$ \\
\hline
\end{tabular}

Fuente: Departamento de estadística del Hospital General Martin Icaza. Elaborado por: López D. Madelyne V. y Cabrera B. Hugo F.

Los resultados muestran que de los pacientes estudiados el mayor número de casos de pancreatitis aguda en pacientes con litiasis biliar se presento a los 20-25 años con el $36 \%$ que representa a 11 pacientes, seguido del $23 \%$ que representa a 7 pacientes entre 46 a 50 años, un 19\% que representa a 6 pacientes entre 26 a 30 años, el 13\% que representa a 4 pacientes entre 31 a 35 años, el $6 \%$ que representa a 2 pacientes entre 41 a 45 años y el menor número de casos con un $3 \%$ que representa a 1 paciente entre 36 a - 40 años. 
Tabla 2. Pancreatitis aguda según el género.

\begin{tabular}{ccc}
\hline \multicolumn{3}{c}{ Pancreatitis aguda según el género } \\
\hline \hline GENERO & \# DE CASOS & PORCENTAJE \\
\hline Masculino & 11 & $35 \%$ \\
\hline Femenino & 20 & $65 \%$ \\
\hline \hline Total & 31 & $100 \%$ \\
\hline
\end{tabular}

Fuente: Departamento de estadística del Hospital General Martin Icaza. Elaborado por:

¿ López D. Madelyne V. y Cabrera B. Hugo F.

El El 65\% de pacientes con litiasis biliar que desarrollaron pancreatitis aguda eran mujeres mientras que el 35\% restante de la población estudiada era hombre.

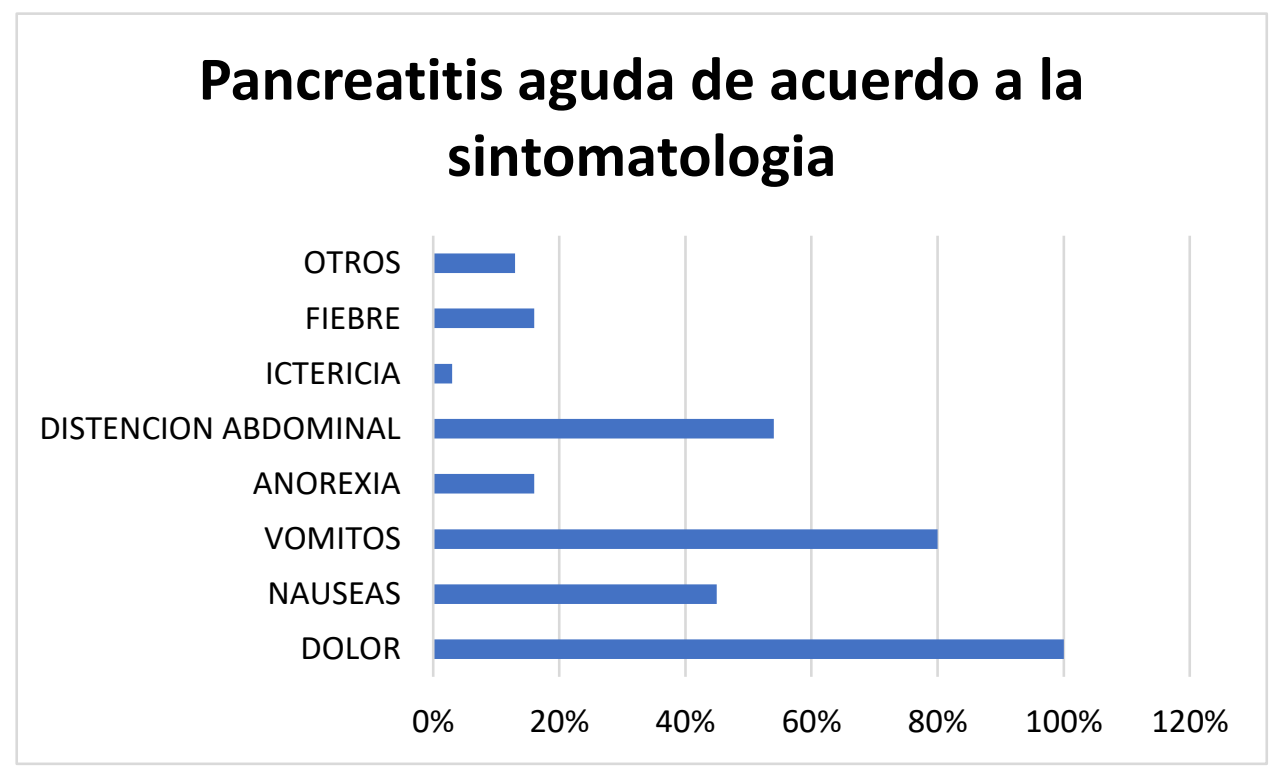

Figura 1. Características clínicas de los pacientes con pancreatitis aguda al momento del ingreso hospitalario.

Fuente: Departamento de estadística del Hospital General Martin Icaza. Elaborado por: López D. Madelyne V. y Cabrera B. Hugo F.

Al identificar la característica clínica de los pacientes que ingresan con pancreatitis aguda se determina que el $100 \%$ de los pacientes acuden a la unidad hospitalaria por presentar dolor abdominal, el $80 \%$ por vomito, el $54 \%$ por distensión abdominal, el $45 \%$ por nauseas, el $16 \%$ por fiebre, el $16 \%$ anorexia, el $13 \%$ por otra sintomatología y el $3 \%$ por ictericia. 


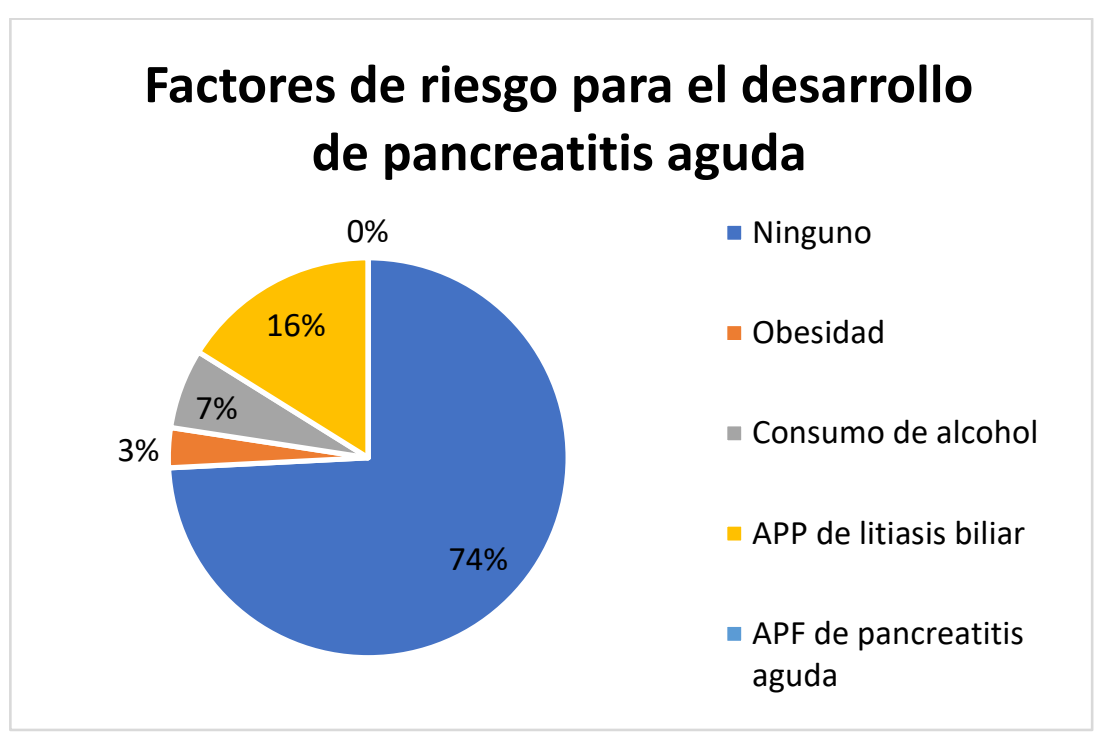

Figura 2. Factores de riesgo para el desarrollo de pancreatitis aguda.

Fuente: Departamento de estadística del Hospital General Martin Icaza. Elaborado por: López D. Madelyne V. y Cabrera B. Hugo F.

Así también analizar los factores de riesgo en el desarrollo de pancreatitis aguda en pacientes con litiasis biliar se puede observar que el $74 \%$ de pacientes no presentaba ningún factor de riesgo a su ingreso, el 16\% presentaba antecedentes patológicos personales de litiasis biliar, mientras que el $7 \%$ tenía antecedentes de alcoholismo y el 3\% de obesidad.

Se observó que el mayor porcentaje de pacientes que padecieron la enfermedad pertenece a la población 20-25 años con un $36 \%$; seguido del $23 \%$ que representa a pacientes entre los 46 a 50 años de edad siendo mas común en el sexo femenino en un $65 \%$.

En un estudio de prevalencia de pancreatitis en adultos jóvenes del año 2016-2017 realizado en el Hospital León Becerra de la ciudad de Milagro; se observó que el $87 \%$ de pancreatitis aguda se presentó en el sexo femenino; con mayor frecuencia en edades entre 28 a 32 años (40\%). En la investigación presente se encontró que un $65 \%$ de las pancreatitis agudas se presentan en el sexo femenino; con mayor frecuencia en edades entre 20 a 25 años de edad (36\%). El 35\% representa el desarrollo de pancreatitis aguda en el sexo masculino. (Chica Salazar, 2018)

Respecto al factor de riesgo se encontró que el $16 \%$ presentaba antecedentes de litiasis biliar al momento de su ingreso; mientras, que el $74 \%$ de pacientes niegan tener algún antecedente o factor de riesgo, cabe recalcar que dentro de este porcentaje queda un margen desconocido de pacientes que al ingreso fueron diagnosticados con pancreatitis aguda precedida por una litiasis biliar que desconocían que presentaban. El 6\% representan los pacientes que tienen hábitos alcohólicos. Parra y Freire (2018) n su estudio de Incidencia y metodología diagnóstica en pacientes con pancreatitis aguda observó que el 53\% presenta antecedente de litiasis biliar en el Hospital Liborio Panchana durante el año 2018. El consumo de alcohol cuenta como factor etiológico en un 39\%; mientras que en el estudio de Chica (2018) la etiología biliar representa un $43,7 \%$.

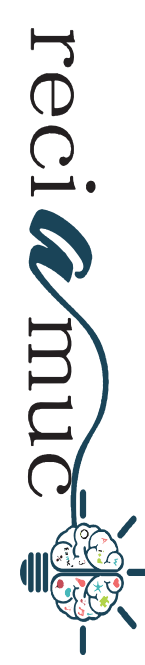


En este estudio encontramos que el dolor abdominal es la manifestación clínica principal que presentan los pacientes con pancreatitis aguda de origen biliar, seguido por vómitos y nauseas. Molina (2018) en su estudio de "Pancreatitis aguda con Coledocolitiasis" determinó que los signos y síntomas con el que debutan los pacientes con pancreatitis aguda de origen biliar es el dolor abdominal (100\%) encontrándose en todos los casos seguido de las náuseas y los vómitos.

Finalmente, este estudio fue realizado en una sola unidad hospitalaria con una muestra considerable de pacientes; lo que nos impide, evaluar la realidad a nivel nacional y analizar las complicaciones posteriores al desarrollo de la enfermedad; por lo que se recomienda la evaluación de más estudios que tengan en cuenta estas variables en esta enfermedad.

\section{Conclusiones}

La pancreatitis aguda se presenta como una enfermedad muy frecuente que produce diferentes Manifestaciones clínicas, fácilmente confusas con otras patologías de tipo digestivas. El objetivo general planteado en este estudio se establece al observar una creciente de casos de pancreatitis aguda en la emergencia de una casa de salud de segundo nivel.

En base a este estudio podemos concluir que el síntoma más característico y el motivo por el cual los pacientes acuden a la emergencia es el dolor abdominal en un $100 \%$, precedido de vómitos $(80 \%)$ y nauseas ( $45 \%$ ) y que las edades más frecuentes en desarrollar una pancreatitis aguda oscila entre los 20 a 25 años de edad y que el sexo más predominante en desarrollar pancreatitis aguda es el sexo femenino, esto puede deberse a varios factores que presenta cada paciente y que la mayoría desconoce; como se demuestra en este estudio, entre los factores de riesgo encontrados tenenos que el $74 \%$ de pacientes niegan presentar algún antecedente patológico personal al momento del ingreso, y que durante su estancia hospitalaria al realizarse diferentes tipos de exámenes, se les diagnóstica que su cuadro es producido por una litiasis biliar; mientras, que el $16 \%$ de pacientes presentaban antecedentes patológicos personales de litiasis biliar antes de su ingreso por pancreatitis aguda.

\section{Bibliografía}

Brito, M. (2002). Pancreatitis aguda. En J. Sanchez, Libro texto de Cirugía. Sociedad Ecuatoriana de Cirugía.

Chica Salazar, D. (2018). Prevalencia de pancreatitis aguda en adultos jóvenes, periodo 2015-2017, en hospital León Becerra. Guayaquil: Universidad de Guayaquil.

Frossard, J., Steer, M., \& Pastor, C. (march de 2008). Antibiotics in acute necrotising pancreatitis-Authors' reply. The Lancet, 371(9618), 143-52.

Instituto Nacional de Estadísticas. (2019). Egresos hospitalarios por condición al egreso y sexo, tasa de letalidad hospitalaria, según grupos de causa de morbilidad. En D. D. SOCIODEMOGRÁFICAS, \& M. Herrera (Ed.), ANUARIO DE ESTADÍSTICAS DE SALUD: CAMAS Y EGRESOS HOSPITALARIOS 2019 (pág. libro 3.1.9).

Jean-Louis Frossard 1, M. L. (junio 12 de 2008). pancreatitis aguda. pubmed.gov, 371 (9607).

Junquera, R., \& Pereyra, I. (enero de 2010). Pancreatitis aguda. Archivos de salud publica, 1(1), 24-30.

Ledesma-Heyer, J. P., \& Arias Amaral, J. (2009). Pancreatitis aguda. Medicina interna de México, 25(4). Recuperado el 12 de mayo de 2021, de medigraphic: https://www.medigraphic.com/pdfs/ medintmex/mim-2009/mim094f.pdf

Molina Gaibor, A. (2018). Pancreatitis aguda con coledocolitiasis julio 2016-julio 2017. Guayaquil: Universidad de Guayaquil. Facultad de Ciencias Médicas.

Parra Ortiz, W. S., \& Freire Jiménez, W. H. (2018). Incidencia y metodología diagnóstica en pacientes con pancreatitis aguda en el Hospital Liborio Panchana. Guayaquil: Universidad de Guayaquil. Facultad de Ciencias Médicas.

Swaroop, V., Chari, S., \& Clain, J. (2004). severe acute pancreatitis. jama, 291(23):2865-8(2865-8).

Toouli, J., Brooke-Smith, M., \& Bassi, C. e. (2002). Guidelines for the management of acute pancreatitis. Gastroenterol Hepatol, 17(S1), 15-39. 


\section{CitAR ESTE ARTICULO:}

López Díaz, M. V., Cabrera Bernal, H. F., Viteri Rojas, A. M., \& Reyes Prieto Aguayo, M. P. (2022). Pancreatitis aguda en pacientes con litiasis biliar ingresados en el hospital Martín Icaza. RECIAMUC, 6(1), 64-71. https://doi. org/10.26820/reciamuc/6.(1).enero.2022.64-71 (c) (1) (\$) (2) BY NC SA

CREATIVE COMMONS RECONOCIMIENTO-NOCOMERCIAL-COMPARTIRIGUAL 4.0 . 